\title{
Elimination of paternal mitochondria through the lysosomal degradation pathway in C. elegans
}

\author{
Qinghua Zhou ${ }^{1}$, Haimin $\mathrm{Li}^{1}$, Ding Xue \\ ${ }^{I}$ Department of Molecular, Cellular, and Developmental Biology, University of Colorado, Boulder, CO 80309, USA
}

In mammals, the inheritance of mitochondrion and its DNA (mtDNA) is strictly maternal, despite the fact that a sperm can inject up to 100 functional mitochondria into the oocyte during fertilization. The mechanisms responsible for the elimination of the paternal mitochondria remain largely unknown. We report here that this paternal mitochondrial elimination process is conserved in Caenorhabditis elegans, and that the lysosomal pathway actively participates in this process. Molecular and cell biological analyses indicate that in wild-type animals paternal mitochondria and mtDNA are destroyed within two hours after fertilization. In animals with compromised lysosomes, paternal mitochondria persist until late embryonic stages. Therefore, the lysosomal pathway plays an important role in degrading paternal mitochondria introduced into the oocyte during fertilization. Our study indicates that $C$. elegans is an excellent animal model for understanding and dissecting this conserved biological process critical for animal development and reproduction.

Keywords: lysosomal degradation; paternal mitochondria elimination; C. elegans; maternal inheritance; mitochondria DNA; fertilized oocyte

Cell Research (2011) 21:1662-1669. doi:10.1038/cr.2011.182; published online 22 November 2011

\section{Introduction}

The mitochondrion is a membrane-enclosed organelle that carries out oxidative phosphorylation to generate a constant supply of adenosine triphosphate (ATP) in eukaryotic cells [1]. It is also the only organelle that contains its own genome (mtDNA) in animals, which encodes multiple protein components of the oxidative phosphorylation complexes I, III, IV, and V [1, 2]. In addition to being an ATP-producing organelle, the mitochondrion also plays critical roles in regulating other cellular processes, including apoptosis [3].

It has been a paradox in development that mammals inherit their mitochondria exclusively from their female parents, despite the fact that the fertilizing spermatozoon could introduce up to 100 mitochondria into the oocyte at the moment of fertilization $[4,5]$. Several different theories have been proposed to account for this observa-

Correspondence: Ding Xue

E-mail: ding.xue@colorado.edu

Received 17 October 2011; accepted 31 October 2011; published online 22 November 2011 tion, including dilution or active destruction of paternal mitochondria in the dividing embryo $[6,7]$, restricted localization of paternal mitochondria to a region of the embryo, or even denial of their entry into the oocyte [8]. It is unclear whether specific elimination of paternal mitochondria is a conserved developmental process in the animal kingdom and how it is regulated and executed [9].

Lysosomes are the major digestive organelles in the cell, responsible for degrading phagocytosed microorganisms or cells and endocytosed macromolecules such as proteins, nucleic acids, and lipids [10]. It also recycles cellular debris, such as excess or obsolete organelles, in a process called autophagy [11]. Lysosomes contain many types of hydrolytic enzymes, including proteases, nucleases, glycosidases, and sulfatases [10], all of which are acid hydrolases with optimal activities in a low $\mathrm{pH}$ environment provided by lysosomes. Therefore, neutralizing this acidity using lysosomotropic agents such as $\mathrm{NH}_{4} \mathrm{Cl}$ can compromise lysosomes' digestion ability $[6,10]$. If paternal mitochondria are actively destructed in fertilized embryos, lysosomes and the autophagy process, with the capability of degrading obsolete organelles, could potentially be involved.

In this study, we developed two complementary meth- 
ods based on polymerase chain reaction (PCR) amplification of mtDNA and time-lapse microscopy imaging of mitochondria stained by fluorescence dyes to monitor the fate of paternal mitochondria in dividing C. elegans embryos. We found that paternal mitochondria and mtDNA were eliminated early during embryogenesis, after the embryos passed the 64-cell stage, and that compromising the lysosomal functions, either by lysosome inhibitor $\mathrm{NH}_{4} \mathrm{Cl}$ or gene knockdown, caused the retention of paternal mitochondria and mtDNA until the late embryonic stages. Moreover, RNAi knockdown of proteasome ubiquitin receptors or autophagy genes also caused delayed removal of paternal mitochondria, suggesting that the ubiquitin-proteasome degradation pathway and the autophagy process may contribute to elimination of paternal mitochondria. These findings indicate that maternal inheritance of mitochondria is conserved in C. elegans and that multiple degradation events may be involved in eliminating paternal mitochondria injected into the fertilized oocyte.

\section{Results}

Development of two complementary assays to track paternal mitochondria and mtDNA in dividing C. elegans embryos

In order to monitor the fate of paternal mitochondria in dividing $C$. elegans embryos, we took advantage of an existing mtDNA allele, $u a D f 5$, which is a $3053 \mathrm{bp}$ deletion in the mtDNA genome and can be detected by PCR analysis (Figure 1A) [12]. When $C$. elegans males heteroplasmic for $u a D f 5$ ( $u a D f 5 /+$ ) and carrying an integrated $\mathrm{P}_{\text {sur-s }}$ GFP transgene (smIs13) were mated with wild-type (N2) hermaphrodites, individual fluorescent
smIs $13 /+$ cross progeny at various embryonic stages from 1.5-fold to 4-fold embryos were screened for the presence of $u a D f 5$ by PCR and all were negative (Figure 1B, lanes 3-4; data not shown). Because from serial dilutions of mtDNA samples, we could detect as few as five copies of $u a D f 5 \mathrm{mtDNA}$ by PCR (Figure 1C), we conclude that the paternal mtDNA was indeed absent in these embryos. In contrast, when we mated smIs 13 males with $u a D f 5 /+$ hermaphrodites, all fluorescent smIs $13 /+$ cross-progeny at various embryonic, larval and adult stages carried uaDf5 (Figure 1B, lanes 1-2; data not shown), indicating that mitochondria are maternally inherited and that the phenomenon of paternal mitochondria elimination is conserved in C. elegans.

We next examined the fate of spermatozoon mitochondria in C. elegans fertilized oocytes using time-lapse fluorescent microscopy. uaDf5/+ males were pre-stained with Mito-Tracker Red (MTR), a mitochondrial specific dye, and then mated with unstained N2 hermaphrodites. Fertilized eggs were dissected out from the mated hermaphrodites and observed using time-lapse fluorescent microscopy. A cluster of red mitochondrial staining was first observed near the site of sperm entry in the fertilized oocyte (Figure 2A), which quickly spread to the whole oocyte as two pronuclei were moving toward each other (Figure 2B and 2C, Supplementary information, Movie S1). This observation clearly indicates that the paternal mitochondria are not denied entry into the oocyte in $C$. elegans, nor are they sequestered to a specific region of the fertilized oocyte.

\section{The timing of paternal mitochondria elimination}

We then determined when the paternal mitochondria were eliminated from the embryo using both PCR and
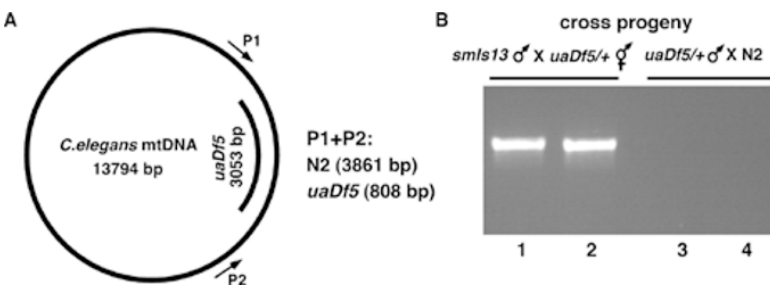

c

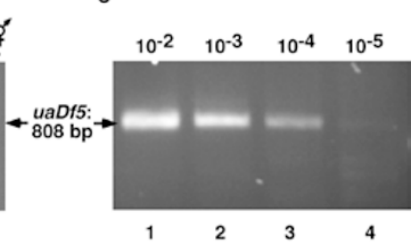

Figure 1 A PCR-based assay to monitor elimination of paternal mitochondria in fertilized oocytes. (A) A schematic diagram of the $C$. elegans mitochondrial genome and the $3053 \mathrm{bp}$ uaDf5 deletion. The positions of the primers used to amplify the uaDf5 deletion and the expected sizes of PCR products from wild-type (N2) and uaDf5 mtDNAs are indicated. (B) C. elegans mitochondria are maternally inherited. 4-fold embryonic stage cross progeny derived from sm/s13 males mated with uaDf5/+ hermaphrodites (lanes 1 and 2), or sm/s13; uaDf5/+ males mated with N2 hermaphrodites (lanes 3 and 4), were examined by PCR for the presence of uaDf5 mtDNA. The expected $3861 \mathrm{bp} \mathrm{PCR}$ product from wild-type mtDNA is not detected under the PCR conditions used. (C) PCR analysis of samples derived from serial dilutions of worm lysate from 10 adult $u a D f 5 /+$ males. The magnitudes of dilution represent the fractions of one worm. Because one uaDf5/+ male has approximately $5.0 \times 10^{5}$ copies of mitochondria [12], we conclude that the PCR protocol could detect as few as five copies of mtDNA (lane 4). 

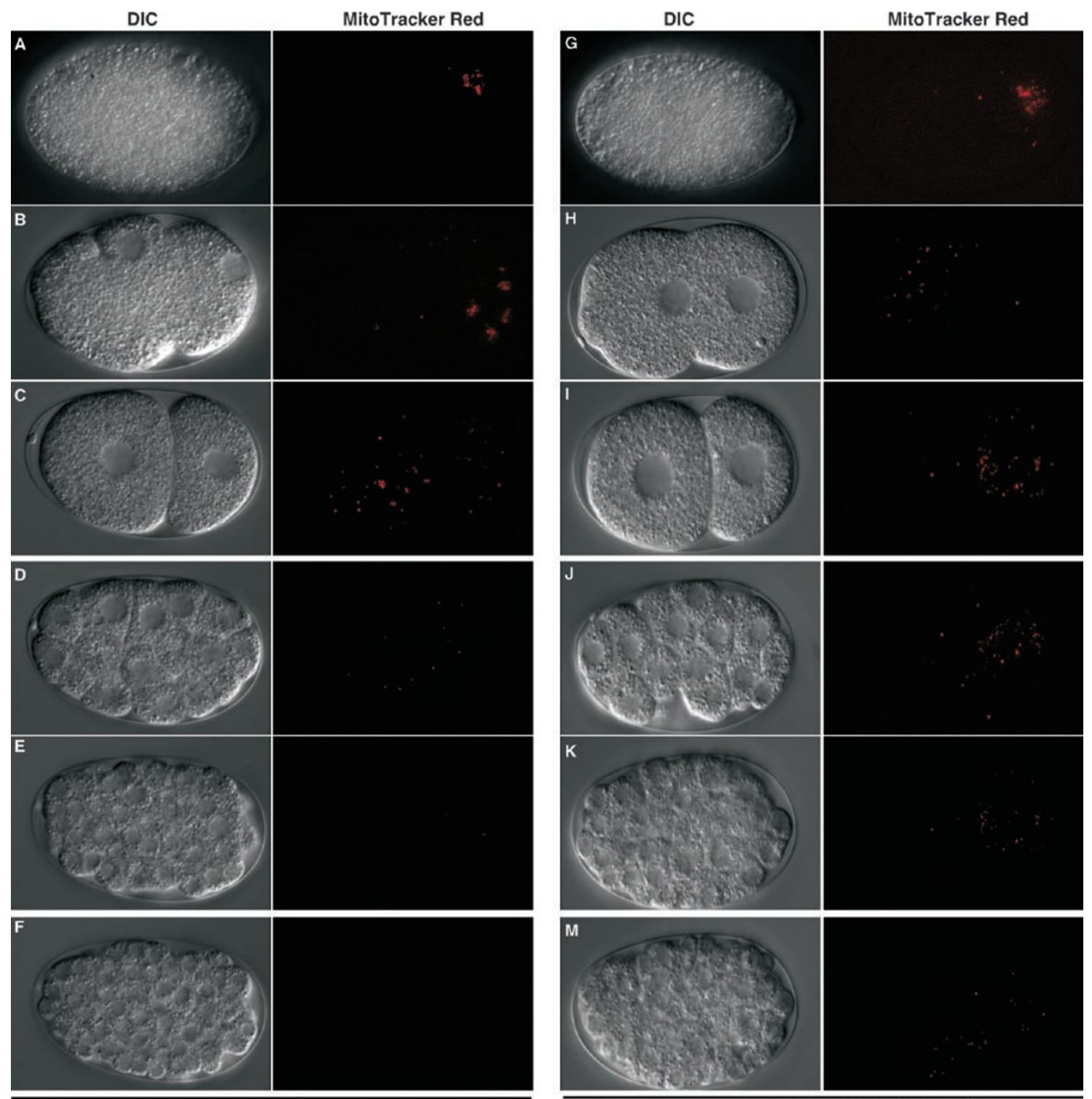

cross progeny from $u a D 15 /+\mathrm{O}^{\prime} \times \mathrm{N} 2$ ?

cross progeny from glo- $1 ;$ uaDf5/+ of $x$ glo-1 ơ

Figure 2 Delayed paternal mitochondria elimination by inhibition of the lysosomal pathway in C. elegans. uaDf5/+ males (left panel) or glo-1(zu391); uaDf5/+ males (right panel) were pre-stained with Mitotracker Red before they were mated with N2 hermaphrodites (left panel) or glo-1(zu391) hermaphrodites (right panel). Fertilized oocytes or embryos were dissected out of the mated hermaphrodites and examined using a fluorescent Nomarski microscope. Differential contrast interference (DIC) and Rhodamine images of the oocytes or embryos are shown. The red dots are mitochondria stained with MitoTracker Red. The stages of the oocyte or embryo are: an oocyte right after the sperm entry $(\mathbf{A}, \mathbf{G})$, an oocyte right before pronucleus fusion (B, H), 2-cell stage embryo (C, I), 32-cell stage embryo (D, J), over-100-cell stage embryo (E, K), and pre-comma stage embryo (F, M).

fluorescent microscopy analyses. In fertilized oocytes before pronucleus fusion and in 1-cell, 4-cell, 16-cell, or 64-cell stage embryos, both MTR-stained mitochondria and $u a D f 5$ mtDNA could be detected (Figure 2B-2D and Figure $3 \mathrm{~B}$, upper panel), but the intensity of staining and PCR signals gradually decreased. Quantification of MTR fluorescent dots in dividing embryos $(n=3)$, which re- flect relative mitochondria numbers, also revealed a gradual decrease in paternal mitochondria numbers (Figure 4). In embryos passing the 64-cell stage, uaDf5 mtDNA was no longer detected by PCR (Figure 3B, upper panel) and mitochondria staining became invisible (Figures 2E, 2F, 4, Supplementary information, Movie S1). In contrast, the intensity of the PCR band specific to the maternal 
A

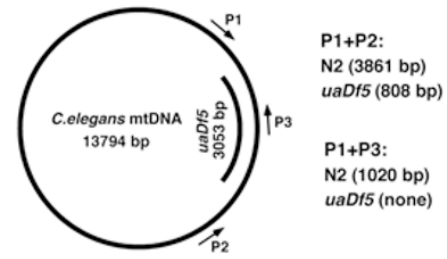

B
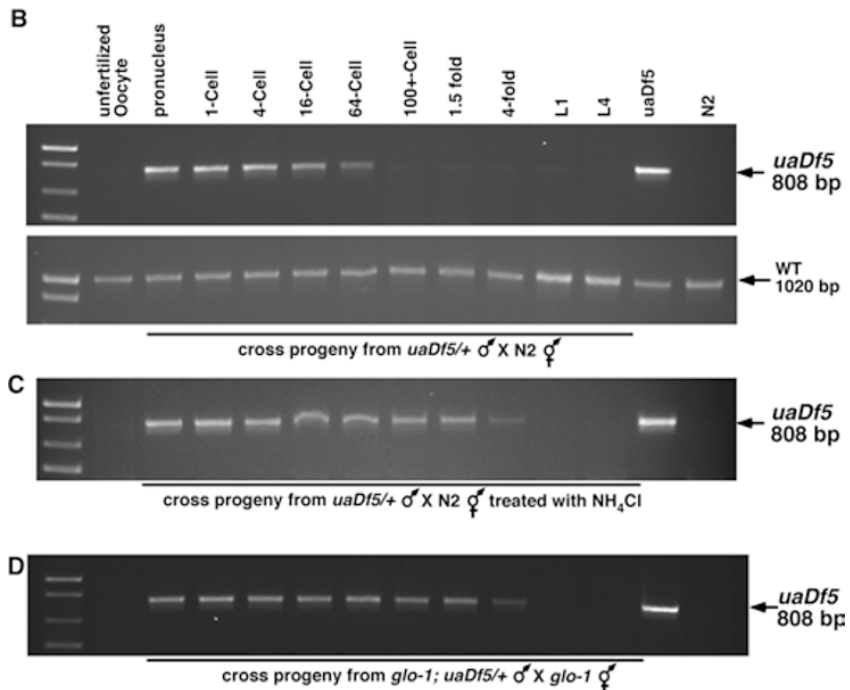

Figure 3 Inhibition of the lysomomal pathway delays paternal mtDNA elimination. (A) A schematic diagram of the $C$. elegans mitochondria genome and the 3053 bp uaDf5 deletion. The positions of the primers used to amplify the uaDf5 deletion and the wild-type mtDNA as well as the expected sizes of PCR products are indicated. (B) Elimination of paternal mtDNA and retention of maternal mtDNA during $C$. elegans development. sm/s 13; uaDf5/+ males were pre-stained with Mitotracker Red before they were mated with N2 hermaphrodites. Single unfertilized oocyte or fertilized egg was dissected out of the mated hermaphrodites and subjected to PCR analysis to detect the presence of the uaDf5 deletion (upper panel) and wildtype mtDNA (bottom panel). (C, D) Inhibition of the Iysomomal pathway delays paternal mtDNA elimination. sm/s13; uaDf5/+ males (C) or sm/s13; glo-1(zu391); uaDf5/+ males (D) were prestained with Mitotracker Red before they were mated with N2 hermaphrodites (C) or glo-1(zu391) hermaphrodites (D). Single unfertilized oocyte or fertilized egg was dissected out of the mated hermaphrodites and subjected to PCR analysis to detect the presence of $u a D f 5 \mathrm{mtDNA}$. In (C) the mating was conducted on plates with $20 \mathrm{mM} \mathrm{NH}_{4} \mathrm{Cl}$. The stages of the oocyte, embryo or larva subjected to PCR analysis are: 1, unfertilized oocyte; 2, fertilized oocyte before pronucleus fusion; 3, 1-cell stage; 4, 4-cell stage; 5, 16-cell stage; 6, 64-cell stage; 7, over-100-cell stage; 8, 1.5-fold stage; 9, 4-fold stage; 10, L1 stage larva; 11, L4 stage larva; 12, uaDf5/+ positive control; 13, N2 control.

mitochondria stayed constant in all embryonic stages and increased in the larval stages (Figure 3A and 3B, bottom panel). These results indicate that paternal mitochondria are probably eliminated in embryos older than the 64-cell stage.

The lysosomal pathway is involved in elimination of paternal mitochondria

Previous studies have implicated that the lysosomal pathway might be involved in eliminating paternal mitochondria [6], because lysosomes are the primary sites for recycling organelles in the cell. We tested whether impairing lysosomal functions in C. elegans could inhibit or delay elimination of paternal mitochondria. Treatment of the mating animals with $20 \mathrm{mM} \mathrm{NH}_{4} \mathrm{Cl}$, a lysosome inhibitor, indeed significantly delayed degradation of paternal mitochondria. We could detect paternal uaDf5 mtDNA by PCR through the 4-fold stage of embryonic development (Figure 3C).

A loss-of-function mutation (zu391) in the glo-1 gene, which encodes a member of the Rab family of small GTPases required for biogenesis of the lysosome-related gut granules [13], results in impaired lysosomal functions and was used to corroborate the role of lysosomes in degrading paternal mitochondria. In cross progeny derived from glo-1(zu391) hermaphrodites mated with smIs 13; glo-1(zu391); uaDf5/+ males, uaDf5 mtDNA was detected by PCR and MTR-stained paternal mitochondria were observed through the 4-fold embryonic stage (Figures

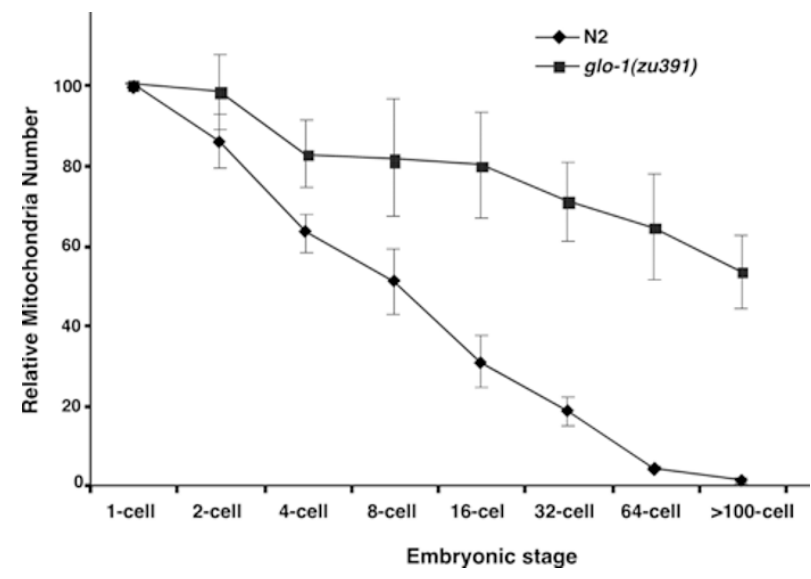

Figure 4 Quantification of relative paternal mitochondria numbers in wild-type or glo-1(zu391) embryos. Mito-Tracker Red fluorescent dots labeled mitochondria clusters and were counted from deconvolved images of the dividing fertilized embryo at different embryonic stages (1-cell, 2-cell, 4-cell, 8-cell, 16-cell, 32-cell, 64-cell, and > 100-cell). The relative paternal mitochondria number in each fertilized oocyte at 1-cell stage was set artificially at 100 . Relative paternal mitochondria numbers in other embryonic stages of the same embryo were normalized with the 1-cell stage number. Three embryos were scored for each strain. Error bars indicate standard deviations (SD). 
A

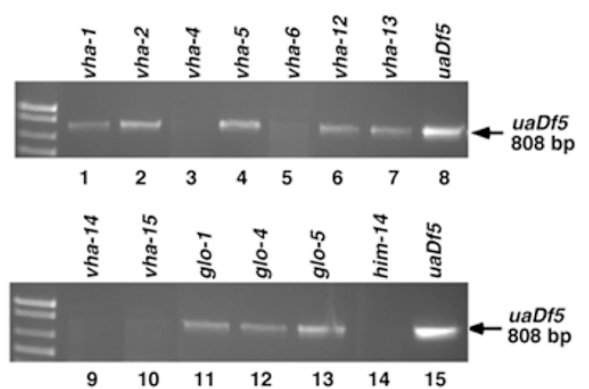

B

\begin{tabular}{clc}
\hline Gene & Molecular Identity & $\begin{array}{c}\text { uaDf5 mtDNA } \\
\text { detected }\end{array}$ \\
\hline vha-1 & Subunit c of the V0 domain of V-ATPase & + \\
vha-2 & Subunit c of the V0 domain of V-ATPase & + \\
vha-4 & Subunit $\mathrm{c}$ of the V0 domain of V-ATPase & + \\
vha-5 & Subunit a of the V0 domain of V-ATPase & + \\
vha-6 & Subunit a of the V0 domain of V-ATPase \\
vha-12 & Subunit B of the V1 domain of V-ATPase & + \\
vha-13 & Subunit A of the V1 domain of V-ATPase & + \\
vha-14 & Subunit D of the V1 domain of V-ATPase \\
vha-15 & Subunit $\mathrm{H}$ of the V1 domain of V-ATPase & + \\
glo-1 & Small GTPase required for lysosome biogenesis \\
glo-4 & Guanine nucleotide exchange factor for GLO-1 & + \\
glo-5 & ABC transporter required for lysosome biogenesis \\
him-14 & RNAi control & + \\
\hline
\end{tabular}

Figure 5 Delayed paternal mtDNA elimination by RNAi knockdown of genes important for the lysosomal functions in $C$. elegans. (A) sm/s13; uaDf5/+ males were mated with N2 hermaphrodites on plates containing bacteria expressing dsRNA from the indicated genes. Multiple GFP-positive sm/s13/+ 4-fold stage embryos were collected and subjected to PCR analysis to detect the presence of uaDf5 mtDNA. him-14 RNAi was used as an RNAi control to demonstrate that RNAi worked (producing higher frequency of males) but was not involved in delaying paternal mtDNA elimination. (B) A summary of the RNAi screen results. The genes undergoing RNAi treatment, their molecular identities, and the effects of RNAi treatments in retaining paternal uaDf5 mtDNA are shown.

2G-2M, 3D, 4, and Supplementary information, Movie S2), confirming that lysosomes are important for removing paternal mitochondria. We further tested the involvement of lysosomes in paternal mitochondria elimination by knocking down a number of genes that are important for lysosomal functions using RNA interference (RNAi). The vacuolar $\mathrm{H}^{+}$-ATPase (V-ATPase) is a universal, multi-subunit proton pump that acidifies intracellular organelles, including lysosomes, and regulates cellular $\mathrm{pH}$ by consuming ATP [14]. RNAi knockdown of vha-12, which encodes a subunit of the V-ATPase required for lysomsome functions [15], resulted in retention of paternal mitochondria until the 4-fold embryonic stage (Figure 5). glo-1, glo-4 (encoding a guanine nucleotide exchange factor for GLO-1), and glo-5 (encoding an ABC transporter), are also required for lysosome biogenesis [13]. RNAi knockdown of these three genes similarly delayed removal of paternal mitochondria until the 4-fold embryonic stage (Figure 5). These results provide further confirmation of the role of lysosomes in the removal of paternal mitochondria.

The autophagy process and the ubiquitin-mediated degradation pathway may be involved in elimination of paternal mitochondria

The involvement of the lysosomal pathway in degrading paternal mitochondria suggests that the autophagy process, which delivers recycled organelles to lysosomes, may be involved [11]. Indeed, RNAi knockdown of four C. elegans autophagy genes, atg-13, bec-1, lgg1 and $\operatorname{lgg}$-3, which are involved in the induction (atg13), vesicle nucleation (bec-1), and vesicle elongation (lgg-1 and lgg-3) steps of the autophagosome formation $[16,17]$, all significantly delayed the removal of paternal mitochondria in fertilized eggs (Figure 6). Therefore, the

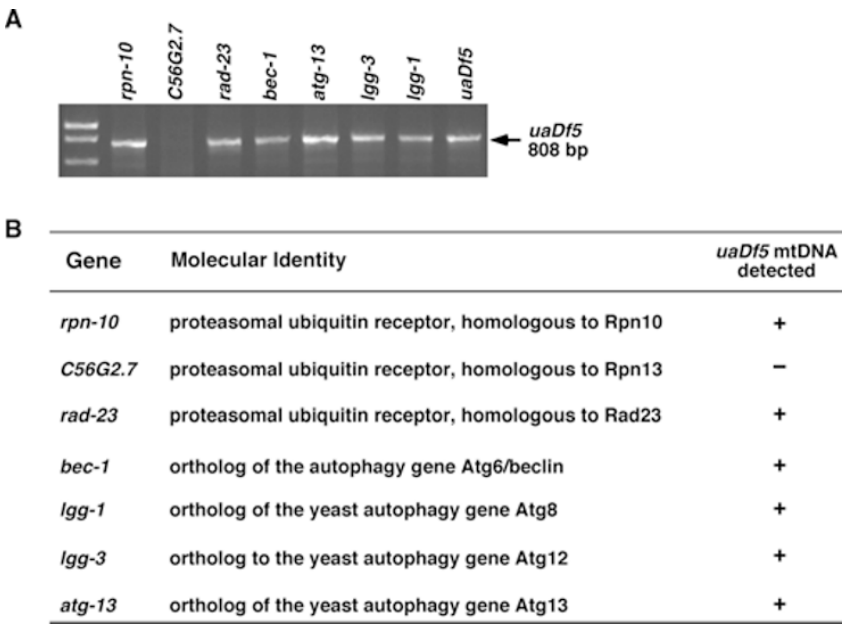

Figure 6 Delayed paternal mtDNA elimination by RNAi knockdown of genes important for autophay and proteasomal degradation in $C$. elegans. (A) $u a D f 5 /+$ males were mated with fem-3(e2006ts) hermaphrodites on plates containing bacteria expressing dsRNA from the indicated genes at $25^{\circ} \mathrm{C}$. fem3 (e2006ts) animals are feminized and sterile at $25^{\circ} \mathrm{C}$ but can mate with males to produce progeny. Multiple 4-fold stage embryos were collected and subjected to PCR analysis to detect the presence of uaDf5 mtDNA. (B) A summary of the RNAi screen results. The genes undergoing RNAi treatment, their molecular identities, and the effects of RNAi treatments in retaining paternal uaDf5 mtDNA are shown. 
autophagy process likely participates in paternal mitochondria elimination upstream of the lysosomal degradation.

Previous studies in mammals also report that sperm mitochondria are ubiquitinated in fertilized oocytes, which may lead to the destruction of sperm mitochondria $[6,18]$. We examined the potential involvement of the ubiquitin degradation pathway in this process by performing RNAi knockdown of three $C$. elegans proteasome ubiquitin receptors, RPN-10, RAD-23, and a homolog of RPN13 (C56G2.7) [19-21]. Proteasomal ubiquitin receptors are known to bind polyubiquitinated substrates and target them to the proteasome for degradation [21]. We found that RNAi treatment of rpn-10 and rad-23, but not C56G2.7, delayed the removal of paternal mitochondria until the 4-fold embryonic stage (Figure 6), suggesting that the ubiquitin-proteasome degradation pathway may also participate in elimination of paternal mitochondria in fertilized eggs.

\section{Discussion}

Elimination of paternal mitochondria is a developmental and reproduction process that is found in most animals $[4,9,22]$. It is not clear how paternal mitochondria are selectively eliminated in fertilized eggs and whether it occurs in the sexual reproduction of invertebrate animals. Several potential mechanisms have been suggested to mediate loss of paternal mitochondria from the fertilized oocytes. These include failed entry of sperm mitochondria into the oocyte, sequestering of paternal mitochondria to a region of the embryo, dilution or active destruction of paternal mitochondria in the dividing embryo, and potentially, both ubiquitin- and lysosomemediated degradation events $[4-7,9,18]$. However, the lack of an appropriate animal model that allows genetic, molecular, and cell biological analyses has prevented rigorous interrogation of this unique cellular process and the various competing theories.

Caenorhabditis elegans is a free-living, transparent nematode that is amenable to genetic, molecular, and cell biological analyses [23]. The invariant cell division patterns of fertilized oocytes and the transparent nature of the embryos are ideal for tracking the fate of paternal mitochondria after the fertilization event [24]. The powerful genetic tools in C. elegans also enable dissection of the molecular mechanisms and components involved in paternal mitochondria elimination. In this study, we report two complementary molecular and cell biological techniques that allow systematic examination of the elimination process of paternal mitochondria in live $C$. elegans animals, starting from the sperm entry into the oocyte to the embryonic stage when paternal mitochondria are eliminated. Our study demonstrates that paternal mitochondria elimination is a conserved process in the sexual reproduction of $C$. elegans. Moreover, elimination of paternal mitochondria is not due to dilution, denied entry, or specific sequestering of spermatozoon mitochondria in fertilized oocytes. Rather, our genetic, molecular and cell biological analyses indicate that the lysosomal pathway is actively involved in degradation of paternal mitochondria. Furthermore, our RNAi knockdown experiments suggest that both the autophagy/lysosomal degradation pathway and the ubiquitin-proteasome degradation pathway are involved in eliminating paternal mitochondria after fertilization, which is consistent with previous observations in mammals $[6,18]$ and two very recent reports that the autophagy process is important for paternal mitochondria elimination in C. elegans $[25,26]$. It will be of great interests to investigate how the autophagy/lysosomal and proteasomal degradation pathways promote removal of paternal mitochondria and whether these two pathways act cooperatively or in parallel to execute this unique cellular event.

Why are paternal mitochondria selectively destructed in fertilized eggs? It has been proposed that in anisogamous fertilization many sperms compete to be the one that fertilizes the oocyte, and in doing so, consume a great deal of energy produced by mitochondria via oxidative phosphorylation [9]. The reactive oxygen species generated during this process could damage the integrity of mtDNA, which in combination with the near absence of DNA repair mechanisms in mitochondria could cause mtDNA to mutate at a faster rate than nuclear DNA [27]. Consistent with this hypothesis, mtDNA from sperms is reported to have a higher mutation rate than that from the oocytes [28]. Therefore, failure to remove paternal mitochondria carrying mutations in mtDNA could result in replication of mutant mitochondria at the expense of maternal mitochondria and compromise the overall fitness of the animals [29]. Indeed, a case report of a patient reveals that failure to eliminate paternal mitochondria in the muscles resulted in a metabolic disorder, which caused a lifelong inability to exercise [30]. Moreover, failure to remove mutated mtDNA contributed by spermatozoon can result in inheritance of mutant paternal mitochondria to the next generation, which could lead to accumulation of mutations in mtDNA after many generations. This presents an evolutionary disadvantage that eventually can lead to extinction of the animals or organisms defective in paternal mitochondria elimination. The C. elegans animal model potentially can be used to interrogate the evolutional and physiological significances of this reproductive process. 


\section{Conclusion}

With the techniques that we develop here to visualize and monitor paternal mitochondria during development in live animals and multiple powerful tools available in C. elegans, it is now possible to identify and dissect components and mechanisms that promote paternal mitochondria elimination. These include the signals that mark sperm mitochondria for destruction and the signaling pathways that recognize and engage the signals to activate the maternal degradation machinery involved in removal of paternal mitochondria. Understanding these important questions will be critical for treating various inherited mitochondrial disease and for determining the safety and efficiency of animal cloning involving spermatid or sperm microinjection.

\section{Materials and Methods}

\section{Strains}

C. elegans strains were maintained at $20{ }^{\circ} \mathrm{C}$ using standard methods [23]. The alleles used for this study are: LGI, smIs 13; LGV, him-5(e1490); LGX, glo-1(zu391); mtDNA, uaDf5 [12, 13]. The N2 Bristol strain was used as the wild-type strain.

\section{RNAi experiments}

Bacteria expressing dsRNA from various candidate genes were obtained from a C. elegans RNAi library (constructed by the Ahringer laboratory) and used to treat mating C. elegans animals using a bacterial feeding protocol [31]. For RNAi of vha genes, L1 larvae were treated with RNAi until they reached the L4 larval stage, when they were moved to a new RNAi plate for the mating experiments. For RNAi of glo genes, L1 larvae were treated with RNAi for one generation before their L4 larval progeny were moved to a new RNAi plate for the mating experiments. The 4-fold stage embryonic cross progeny from the above mating experiments were then used for PCR analyses to detect the presence of $u a D f 5$ deletion.

\section{Drug treatment}

In $\mathrm{NH}_{4} \mathrm{Cl}$ treatment experiments, $2 \mathrm{M} \mathrm{NH}_{4} \mathrm{Cl}$ was diluted 100 folds into freshly grown OP50 bacteria, which were spread on the NGM plates. Males and hermaphrodites were mated on these freshly made $\mathrm{NH}_{4} \mathrm{Cl}$-containing plates overnight before the mated hermaphrodites were dissected to obtain cross progeny.

\section{MitoTracker Red staining}

$1 \mathrm{mM}$ MitoTracker Red in Dimethyl Sulphoxide (DMSO) was diluted into the $\mathrm{M} 9$ buffer $\left(42.3 \mathrm{mM} \mathrm{Na}_{2} \mathrm{HPO}_{4}, 22 \mathrm{mM} \mathrm{KH}_{2} \mathrm{PO}_{4}, 8.6\right.$ $\mathrm{mM} \mathrm{NaCl}, 18.7 \mathrm{mM} \mathrm{NH} 4 \mathrm{Cl}$ ) to a final concentration of $50 \mu \mathrm{M}$, which was dropped directly onto L4 larval males placed on an OP50-seeded plate. After $12 \mathrm{~h}$, the stained males were transferred to a new seeded plate with plenty OP50 bacteria for $15 \mathrm{~min}$ to remove the dye that might attach to the worm body (we called this step "bacterial shower"). After three "bacterial showers", stained males were mated with unstained young adult hermaphrodites for $8 \mathrm{~h}$ before the mated hermaphrodites were dissected to obtain cross progeny.

\section{Four-dimensional fluorescent microscopy}

Four-dimensional microscopy analysis was performed as described previously [32]. Briefly, after the mated hermaphrodite was dissected and embryos were pushed out of the dissected hermaphrodite on an agarose pad, a coverslip was applied on to the embryos and egg salt buffer $(118 \mathrm{mM} \mathrm{NaCl}$ and $48 \mathrm{mM} \mathrm{KCl})$ was pipetted into the empty space between the coverslip and the slide. The sample was then sealed with a hot mixture of petrol gel and bee wax. 3-D images were taken from the top to the bottom of the embryo in a 20-z series using a Zeiss Axioplan 2 Nomarski microscope equipped with a Cohu CCD camera and SlideBook 5 software (Intelligent Imaging Innovations, Inc.). All fluorescent images were deconvolved and projected into one plane. Each fluorescent image therefore showed almost all MTR-stained mitochondria observed in the embryo.

Quantification of paternal mitochondria in dividing embryos MTR fluorescent dots labeled mitochondria clusters and were counted from deconvolved images of the dividing fertilized embryo at different embryonic stages. The relative paternal mitochondria number for each fertilized egg at 1-cell stage was set artificially at 100 . Relative paternal mitochondria numbers in other embryonic stages of the same embryo were normalized using the 1 -cell stage number as a standard.

\section{Molecular Biology}

Single or multiple fertilized eggs dissected from the mated hermaphrodites were screened for the appropriate embryonic stages under the microscope and subjected to PCR analyses. The primers used to detect the $u a D f 5$ deletion are: P1 5'-GATTAGCACAAGCTTTATTGGATGG-3' and P2 5'-AAGATCTTAACATTCCGGCTGAGGC- ${ }^{\prime}$. The primers used to detect the wild-type mtDNA are: P1 5'-GATTAGCACAAGCTTTATTGGATGG-3' and P3 5'-AAGTACTTAATCACGCTACAG-3'.

\section{Acknowledgments}

We would like to thank Grant Weaver (University of Colorado) for comments on the manuscript and members of the Xue lab for discussions and suggestions. This work is supported by grants from NIH (R01GM59083, R01GM79097 and R01GM088241) and a Burroughs Wellcome Fund Award to DX.

\section{References}

1 Henze K, Martin W. Evolutionary biology: Essence of mitochondria. Nature 2003; 426:127-128.

2 Wallace DC. Mitochondrial DNA mutations in disease and aging. Environ Mol Mutagen 2010; 51:440-450.

3 Wang X. The expanding role of mitochondria in apoptosis. Genes Dev 2001; 15:2922-2933.

4 Hutchison CA 3rd, Newbold JE, Potter SS, Edgell MH. Maternal inheritance of mammalian mitochondrial DNA. Nature 1974; 251:536-538.

5 Giles RE, Blanc H, Cann HM, Wallace DC. Maternal inheri- 
tance of human mitochondrial DNA. Proc Natl Acad Sci USA 1980; 77:6715-6719.

6 Sutovsky P, Moreno RD, Ramalho-Santos J, Dominko T, Simerly C, Schatten G. Ubiquitinated sperm mitochondria, selective proteolysis, and the regulation of mitochondrial inheritance in mammalian embryos. Biol Reprod 2000; 63:582590.

7 Nishimura Y, Yoshinari T, Naruse K, et al. Active digestion of sperm mitochondrial DNA in single living sperm revealed by optical tweezers. Proc Natl Acad Sci USA 2006; 103:13821387.

8 Ankel-Simons F, Cummins JM. Misconceptions about mitochondria and mammalian fertilization: implications for theories on human evolution. Proc Natl Acad Sci USA 1996; 93:13859-13863.

9 Wallace DC. Why do we still have a maternally inherited mitochondrial DNA? Insights from evolutionary medicine. Annu Rev Biochem 2007; 76:781-821.

10 Luzio JP, Pryor PR, Bright NA. Lysosomes: fusion and function. Nat Rev Mol Cell Biol 2007; 8:622-632.

11 Mizushima N, Levine B, Cuervo AM, Klionsky DJ. Autophagy fights disease through cellular self-digestion. Nature 2008; 451:1069-1075.

12 Tsang WY, Lemire BD. Stable heteroplasmy but differential inheritance of a large mitochondrial DNA deletion in nematodes. Biochem Cell Biol 2002; 80:645-654.

13 Hermann GJ, Schroeder LK, Hieb CA, et al. Genetic analysis of lysosomal trafficking in Caenorhabditis elegans. Mol Biol Cell 2005; 16:3273-3288.

14 Nishi T, Forgac M. The vacuolar (H+)-ATPases--nature's most versatile proton pumps. Nat Rev Mol Cell Biol 2002; 3:94-103.

15 Syntichaki P, Samara C, Tavernarakis N. The vacuolar H+ -ATPase mediates intracellular acidification required for neurodegeneration in C. elegans. Curr Biol 2005; 15:1249-1254.

16 Meléndez A, Levine B. Autophagy in C. elegans. WormBook (August 24, 2009), WormBook, ed. The C. elegans Research Community, WormBook, doi/10.1895/wormbook.1.147.1

17 Meléndez A, Tallóczy Z, Seaman M, Eskelinen EL, Hall DH, Levine B. Autophagy genes are essential for dauer development and life-span extension in C. elegans. Science 2003; 301:1387-1391.
18 Sutovsky P, Moreno RD, Ramalho-Santos J, Dominko T, Simerly C, Schatten G. Ubiquitin tag for sperm mitochondria. Nature 1999; 402:371-372.

19 Shimada M, Kanematsu K, Tanaka K, Yokosawa H, Kawahara H. Proteasomal ubiquitin receptor RPN-10 controls sex determination in Caenorhabditis elegans. Mol Biol Cell 2006; 17:5356-5371.

20 Husnjak K, Elsasser S, Zhang N, et al. Proteasome subunit Rpn13 is a novel ubiquitin receptor. Nature 2008; 453:481488.

21 Madura K. Rad23 and Rpn10: perennial wallflowers join the melee. Trends Biochem Sci 2004; 29:637-640.

22 Pakendorf B, Stoneking M. Mitochondrial DNA and human evolution. Annu Rev Genomics Hum Genet 2005; 6:165-183.

23 Brenner S. The genetics of Caenorhabditis elegans. Genetics 1974; 77:71-94.

24 Sulston JE, Schierenberg E, White JG, Thomson JN. The embryonic cell lineage of the nematode Caenorhabditis elegans. Dev Biol 1983; 100:64-119.

25 Sato M, Sato K. Degradation of paternal mitochondria by fertilization-triggered autophagy in C. elegans embryos. Science 2011 Oct 13. doi:10.1126/science. 1210333

26 Al Rawi S, Louvet-Vallee S, Djeddi A, et al. Postfertilization autophagy of sperm organelles prevents paternal mitochondrial DNA transmission. Science 2011 Oct 27. doi:10.1126/ science. 1211878

27 Nachman MW, Brown WM, Stoneking M, Aquadro CF. Nonneutral mitochondrial DNA variation in humans and chimpanzees. Genetics 1996; 142:953-563.

28 Cummins JM. Fertilization and elimination of the paternal mitochondrial genome. Hum Reprod 2000; 2:92-101.

29 Hastings IM. Population genetic aspects of deleterious cytoplasmic genomes and their effect on the evolution of sexual reproduction. Genet Res 1992; 59:215-225.

30 Schwartz M, Vissing J. Paternal inheritance of mitochondrial DNA. N Engl J Med 2002; 347:576-580.

31 Wang X, Yang C, Chai J, Shi Y, Xue D. Mechanisms of AIFmediated apoptotic DNA degradation in Caenorhabditis elegans. Science 2002; 298:1587-1592.

32 Wang X, Wu YC, Fadok VA, et al. Cell corpse engulfment mediated by $C$. elegans phosphatidylserine receptor through CED-5 and CED-12. Science 2003; 302:1563-1566.

(Supplementary information is linked to the online version of the paper on the Cell Research website.) 